\title{
openheart Association of early versus delayed normalisation of left ventricular ejection fraction with mortality in ischemic cardiomyopathy
}

\author{
Walter B Schiffer (D) , Andrew Perry, ${ }^{2}$ Elena Deych, ${ }^{3}$ David L Brown (D) , \\ Luigi Adamo (i) ${ }^{4}$
}

To cite: Schiffer WB, Perry A, Deych E, et al. Association of early versus delayed normalisation of left ventricular ejection fraction with mortality in ischemic cardiomyopathy. Open Heart 2021;8:e001528. doi:10.1136/

openhrt-2020-001528

Received 20 November 2020 Revised 22 December 2020 Accepted 22 February 2021

\section{Check for updates}

(C) Author(s) (or their employer(s)) 2021. Re-use permitted under CC BY-NC. No commercial re-use. See rights and permissions. Published by BMJ.

${ }^{1}$ Internal Medicine, Washington University in St Louis, St Louis, Missouri, USA

${ }^{2}$ Cardiovascular Division, Department of Internal Medicine University of Washington School of Medicine, Seattle, Washington, USA

${ }^{3}$ Cardiovascular Division, Department of Internal Medicine, Washington University in St. Louis, St. Louis, Missouri, USA ${ }^{4}$ Cardiovascular Division, Department of Internal Medicine, The Johns Hopkins University School of Medicine, Baltimore, Maryland, USA

Correspondence to Dr Luigi Adamo; ladamo2@ jhmi.edu

\section{ABSTRACT}

Objective In patients with non-ischaemic cardiomyopathy and reduced left ventricular ejection fraction (LVEF), normalisation of LVEF is associated with improved outcomes. However, data on patients with ischaemic cardiomyopathy and recovered LVEF are lacking. The goal of this study was to assess the prognostic significance of normalisation of the LVEF in patients with ischaemic cardiomyopathy.

Methods/Results We performed a non-prespecified post hoc analysis of the Surgical Treatment for Ischaemic Heart Failure (STICH) trial to determine the association between normalisation of LVEF (>50\%) and mortality during followup. Of the 1212 patients with LVEF $<35 \%$ enroled in the STICH trial, 932 underwent assessment of LVEF at 4 months and/or 2 years after enrolment. Among them, 18 patients experienced normalisation in LVEF at 4-month follow-up and 35 patients experienced recovery in LVEF at 2 years. Recovery of LVEF at 4 months and recovery of LVEF at 2 years were not correlated. Recovery of LVEF at 4 months was not associated with reduced all-cause mortality in unadjusted analysis (log-rank test $p=0.54$ ) or in Cox proportional hazards analysis (HR: $0.93 ; 95 \% \mathrm{Cl}$ : 0.48 to $1.80 ; p=0.82$ ). Ejection fraction recovery at 2 years was associated with a reduction in all-cause mortality, both in unadjusted analysis (log-rank test $p=0.004$ ) and in the Cox proportional hazard model (HR: $0.41 ; 95 \% \mathrm{Cl}: 0.21$ to $0.80 ; p=0.009$ ).

Conclusions In patients with ischaemic cardiomyopathy, delayed normalisation of LVEF is associated with reduced mortality, whereas early recovery of LVEF is not. Further studies are needed to confirm these findings.

\section{INTRODUCTION}

As management strategies for heart failure with reduced ejection fraction (HFrEF) continue to improve, more patients experience improvement in left ventricular ejection fraction (LVEF) to a normal range $(>50 \%)$. Several studies have suggested that even though recovery of LVEF typically does not correspond to a true normalisation of myocardial function, ${ }^{12}$ it is associated with a marked improvement in outcomes. ${ }^{2} 3$ However,

\section{Key questions}

What is already known about this subject?

- Many patients with non-ischaemic cardiomyopathy experience reverse left ventricular remodelling to the point of regaining an ejection fraction in the normal range. This is associated with an improvement in prognosis. In patients with ischaemic cardiomyopathy normalisation of left ventricular ejection fraction (LVEF) is much less common and its prognostic significance is unclear.

What does this study add?

- Through a non-prespecified post-hoc analysis of patients enroled in the Surgical Treatment for Ischaemic Heart Failure trial, we found that patients with ischaemic cardiomyopathy can experience early or delayed normalisation in LVEF. The two events are rare and are not correlated. Delayed normalisation in LVEF is associated with reduced mortality, early normalisation is not.

How might this impact on clinical practice?

- This study indicates that clinicians caring for patients with ischaemic cardiomyopathy and reduced LVEF can interpret delayed normalisation in LVEF as a positive prognostic marker but should interpret early normalisation in LVEF with caution.

these analyses have focused on cohorts with a majority, or exclusively, of patients with heart failure of non-ischaemic aetiology. ${ }^{3-7}$ In the landmark study that introduced the concept of heart failure with recovered LVEF, Basuray et al studied a cohort in which only $16 \%$ of patients had ischaemic cardiomyopathy (ICM). ${ }^{3}$ Other studies that focused on outcomes in patients with normalised LVEF included a percentage of patients with ICM ranging from $0 \%{ }^{67}$ to $35 \% .^{5}$

Epidemiological data suggest that the timing and biology of reverse cardiac remodelling and 'apparent healing' differs depending on the aetiology of heart failure. 
In particular, patients with ICM are less likely to experience normalisation in $\mathrm{LVEF}^{59}$ and they are likely to have focal areas of permanent myocardial damage that are independent predictors of poor outcome. ${ }^{10}$ Therefore, it is unclear whether findings based on cohorts of patients highly enriched in heart failure of non-ischaemic aetiology apply to patients with ICM and recovered LVEF. It also remains unclear what prognostic value a normalised LVEF holds in this patient population.

In order to start filling this gap in knowledge, we analysed data collected in the Surgical Treatment for Ischaemic Heart Failure (STICH) trial. The STICH trial was designed to compare the benefit of surgical revascularisation with medical therapy to that of medical therapy alone in patients with ICM and LVEF $<35 \%{ }^{8}$ Patients were assessed with follow-up echocardiogram at 4 months and 2 years postrandomisation and were followed for 10 years to assess mortality. ${ }^{11} 12$

\section{METHODS \\ Data source}

The STICH dataset was obtained from the NHLBI under a data use agreement. The Washington University Human Research Protection Office granted this study an exemption from Institutional Review Board oversight due to the de-identified nature of the data.

\section{Study population}

The rationale and methodology for patient selection in the STICH trial have been described previously. ${ }^{13}$ In brief, STICH was an international, multicentre, nonblinded, randomised trial. Patients included in the study had coronary artery disease, were eligible for coronary artery bypass graft (CABG) surgery and had a $\mathrm{LVEF} \leq 35 \%$. Patients were then randomised to one of three treatment arms: optimal medical therapy (OMT), CABG plus OMT or CABG plus OMT and surgical ventricular repair. For this study, we analysed only patients randomised to the CABG plus OMT or OMT alone (hypothesis 1).

Of the 1212 patients with LVEF $\leq 35 \%$ enroled in the STICH trial and randomised to OMT or CABG plus OMT, 932 underwent echocardiographic assessment of LVEF at 4 months and/or 2 years, while 587 patients had LVEF assessment at both time points. No assessment was done at other times. A total of 267 subjects without recorded ejection fraction $(\mathrm{EF})$ were excluded. Two subjects with baseline EF $>50 \%$ were also excluded. There were also 11 patients with $\mathrm{EF}$ reported after date of death who were excluded. We defined LVEF recovery as LVEF $>50 \%$. In the STICH trial, LVEF was measured either via visual estimate or biplane method, and measurements obtained with these two methods within this cohort were highly correlated. ${ }^{14}$

\section{Statistical analysis}

Baseline clinical data are represented as median with IQR for continuous variables and as counts with percentages for categorical data. The McNemar test was used to assess the relationship between LVEF recovery at 4 months and LVEF recovery at 2 years. Mortality was compared between patients with recovered LVEF and patients without recovered LVEF at 4 months or 2 years with multivariate Cox proportional hazard models. Because patients in the STICH trial did not have information on recovery status between 4 months and 2 years, and because most patients who recovered at 4 months did not retain their recovery status (described in the Results section), a time to recovery variable could not be accurately calculated. We therefore decided against including the recovery status as a time-dependent variable in the Cox proportional hazard model. Likewise, we could not include 4-month and 2-year recovery as two independent predictors in one model, as a subject would have to be alive at 2 years in order to have an assessment at that time and not all patients alive at 4 months were alive at 2 years. Instead, we assessed early and late recovery separately. For the 4-month recovery model, the time to event was calculated from 4-month status assessment; for the 2-year model, it was calculated starting from 2-year assessment. The proportional hazard assumptions were tested, and appropriate diagnostics were performed. Three patients were censored over the course of long-term follow-up because of missing information. We included variables known to correlate with mortality in patients with ICM as covariates: age, body mass index (BMI), diabetes, sex, randomisation to CABG versus medical therapy and OMT. OMT was defined as therapy with aspirin, statin, beta blocker and ACE inhibitor or angiotensin receptor blocker at 4 months postrandomisation. Because of low frequencies, implanted cardiac defibrillator (ICD) and cardiac resynchronisation therapy (CRT) implantation variables were excluded from the models. The existing literature indicates that in the STICH trial CABG was not independently associated with LVEF improvement but was associated with mortality. ${ }^{15}$ To test whether the effect of LVEF improvement on survival was different for CABG versus medical therapy, we investigated the interaction between therapy type and LVEF recovery. The interaction was insignificant for the 4-month time point and the 2-year time point ( $\mathrm{p}$ values 0.379 and 0.753 , respectively), and therefore was not included in the final models.

\section{RESULTS \\ Patient characteristics}

Of the 1212 patients with $\mathrm{LVEF} \leq 35 \%$ enroled in the STICH trial, 932 underwent assessment of LVEF at 4 months and/or 2 years after enrolment. The 932 patients included in our analysis did not differ significantly from the STICH population not included in the analysis with respect to demographics and randomised treatment assignment to OMT plus CABG or OMT alone, they had slightly higher prevalence of diabetes $(41 \%$ vs $33 \%$, $\mathrm{p}=0.02)$ and slightly lower prevalence of CRT ( $7 \%$ vs $11 \%$, $\mathrm{p}=0.04$, table 1 ). Among them, 18 patients experienced normalisation in LVEF (>50\%) at 4-month follow-up 
Table 1 Comparison between patients with LVEF assessment (included in this study) and patients without LVEF assessment (excluded from this study)

\begin{tabular}{lcccl}
\hline Comorbidity & STICH & Included & Excluded & P value included vs excluded \\
\hline Age, mean (SD) & $60(9)$ & $61(9)$ & $59(9)$ & 0.11 \\
BMI (SD) & $27(5)$ & $27(5)$ & $27(5)$ & 0.06 \\
Medical therapy vs CABG, N (\%) & $590(50)$ & $476(51)$ & $114(45)$ & 0.09 \\
Male, N (\%) & $1042(88)$ & $810(87)$ & $232(91)$ & 0.07 \\
Diabetes, N (\%) & $468(39)$ & $385(41)$ & $83(33)$ & 0.02 \\
CRT (\%) & $92(8 \%)$ & $64(7 \%)$ & $28(11 \%)$ & 0.04 \\
ICD (\%) & $28(2 \%)$ & $24(3 \%)$ & $4(2 \%)$ & 0.49 \\
\hline
\end{tabular}

Data are presented as mean (SD) for continuous variables and number (percentage) for categorical variables. Patients included and excluded from the analysis were compared using t-test for continuous variables and $\chi^{2}$ test for categorical variables.

STICH refers to all patients included in the STICH trial. Included refers to subgroup of STICH trial patients who had LVEF assessment during follow-up and were included in this study. Excluded refers to subgroup of STICH trial patients who did not have LVEF assessment during follow-up and were excluded from this study. Medical therapy versus CABG refers to patients randomised to medical therapy alone or to coronary artery bypass graft plus medical therapy.

BMI, body mass index; CABG, coronary artery bypass graft; CRT, cardiac resynchronisation therapy; ICD, permanent implanted cardiac defibrillator; LVEF, left ventricular ejection fraction; STICH, Surgical Treatment for Ischaemic Heart Failure.

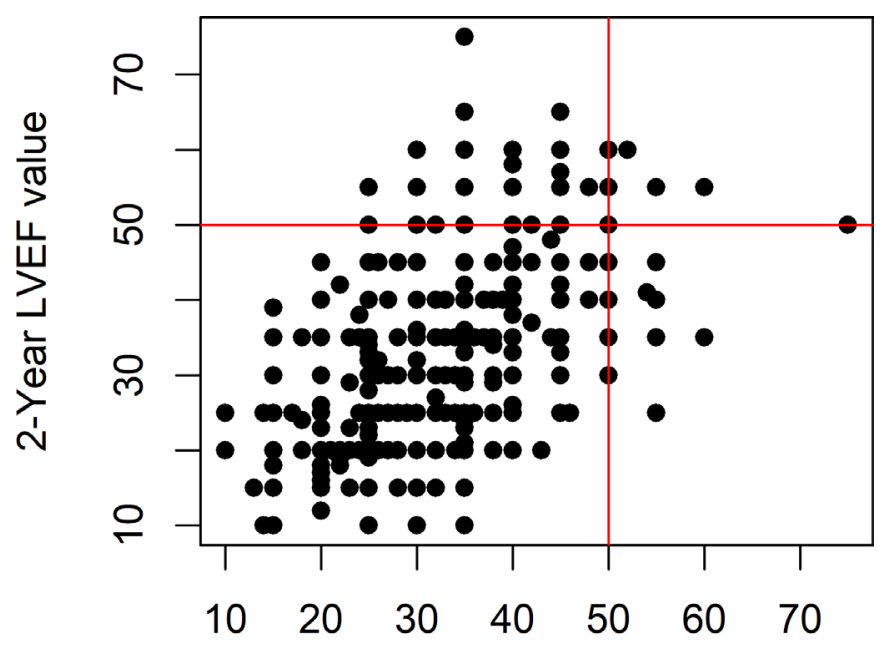

4-Month LVEF value

Figure 1 Relationship between LVEF at 4 months and LVEF at 2 years: each patient with LVEF measurements at both 4 months and 2 years is represented on the plot as a dot. Red lines mark LVEF $=50 \%$ and identify four quadrants. The lower left quadrant includes patients with LVEF $<50 \%$ at both 4 months and 2 years, the upper left quadrant includes patients with LVEF $>50 \%$ at 2 years but $<50 \%$ at 4 months, the upper right quadrant includes patients with LVEF $>50 \%$ at both time points and the lower right quadrant includes patients with LVEF $>50 \%$ at 4 months and LVEF $<50 \%$ at 2 years. The dots are grouped along the diagonal, suggesting correlation between LVEF at 4 months and LVEF at 2 years. However, the number of dots in the right upper quadrant is much lower than the number of dots in the upper left quadrant + lower right quadrant indicating significant disagreement between LVEF recovery at 4 months and LVEF recovery at 2 years $(\mathrm{McNemar}$ test $\mathrm{p}=0.00047)$. LVEF, left ventricular ejection fraction. and 35 patients experienced recovery in LVEF $(>50 \%)$ at 2 years.

Relationship between LVEF recovery at 4 months and LVEF recovery at 2 years

A total of 587 patients had LVEF information at both 4 months and 2 years. There was a weak correlation between LVEF at 4 months and LVEF at 2 years (Spearman correlation $0.56, \mathrm{p}<0.0001)$. However, there was no consistency in LVEF recovery status between 4 months and 2 years (McNemar test $\mathrm{p}=0.0005$, figure 1 ). Of 11 patients who had recovered LVEF at 4 months and had a 2-year LVEF measurement, only three continued to have LVEF $>50 \%$ at 2 years (right upper quadrant in figure 1).

Relationship between LVEF recovery at 4 months and mortality A total of 885 patients had LVEF measurements at 4 months. These 885 patients did not differ significantly from the patients who did not have LVEF measurement at 4 months with respect to demographics, comorbidities and randomisation to OMT plus CABG or OMT alone (online supplemental table 1). Of these 885 patients, 18 were found to have recovered LVEF, while 867 were found to have reduced LVEF. Patients with recovered LVEF at 4 months were similar to patients with reduced LVEF in terms of age, gender, BMI, prevalence of ICDs or CRT and randomisation group within the STICH trial but had a trend towards higher prevalence of diabetes that was statistically insignificant $(66.7 \%$ vs $41.1 \%, \mathrm{p}=0.052$, table 2). LVEF recovery at 4 months was not associated with reduced all-cause mortality during follow-up in unadjusted analysis (Kaplan-Meyer log-rank $\mathrm{p}=0.54$, figure 2) or in the Cox proportional hazards model (HR: 0.93 ; $95 \%$ CI: 0.48 to $1.80 ; \mathrm{p}=0.82$; table 3 ). 
Table 2 Comparison between patients with and without LVEF recovery at 4 months

\begin{tabular}{|c|c|c|c|}
\hline & $\begin{array}{l}\text { LVEF } \leq 50 \% \\
\text { at } 4 \text { months } \\
(n=867)\end{array}$ & $\begin{array}{l}\text { LVEF >50\% } \\
\text { at } 4 \text { months } \\
(n=18)\end{array}$ & $P$ value \\
\hline Age (SD) & 60.57 (9.29) & $60.81(8.02)$ & 0.915 \\
\hline BMI (SD) & $27.43(4.71)$ & $27.93(3.91)$ & 0.654 \\
\hline $\begin{array}{l}\text { Medical therapy vs } \\
\text { CABG (\%) }\end{array}$ & 449 (51.8) & $6(33.3)$ & 0.189 \\
\hline Male gender (\%) & 755 (87.1) & 13 (72.2) & 0.136 \\
\hline Diabetes (\%) & 356 (41.1) & $12(66.7)$ & 0.052 \\
\hline CRT=Yes (\%) & $57(6.6)$ & $3(16.7)$ & 0.117 \\
\hline ОМТ & 567 (65.4) & $12(66.7)$ & 1 \\
\hline ICD & $22(2.5)$ & $0(0.0)$ & 1 \\
\hline
\end{tabular}

Data presented as mean (SD) for continuous variables and number (percentage) for categorical variables. Patients with and without recovered LVEF at 4 months were compared using t-test for continuous variables and $\chi^{2}$ test for categorical variables. Medical therapy versus CABG refers to patients randomised to medical therapy alone or to CABG plus medical therapy. OMT refers to patient on OMT at 4 months follow-up.

$\mathrm{BMI}$, body mass index; CABG, coronary artery bypass graft; CRT, cardiac resynchronisation therapy; ICD, permanent implanted cardiac defibrillator; LVEF, left ventricular ejection fraction; OMT, optimal medical therapy.

Relationship between LVEF recovery at 2 years and mortality A total of 1116 patients survived for at least 2 years. Of these, 634 patients had LVEF measurements at 2 years and were therefore included in the analysis of survival post 2-year

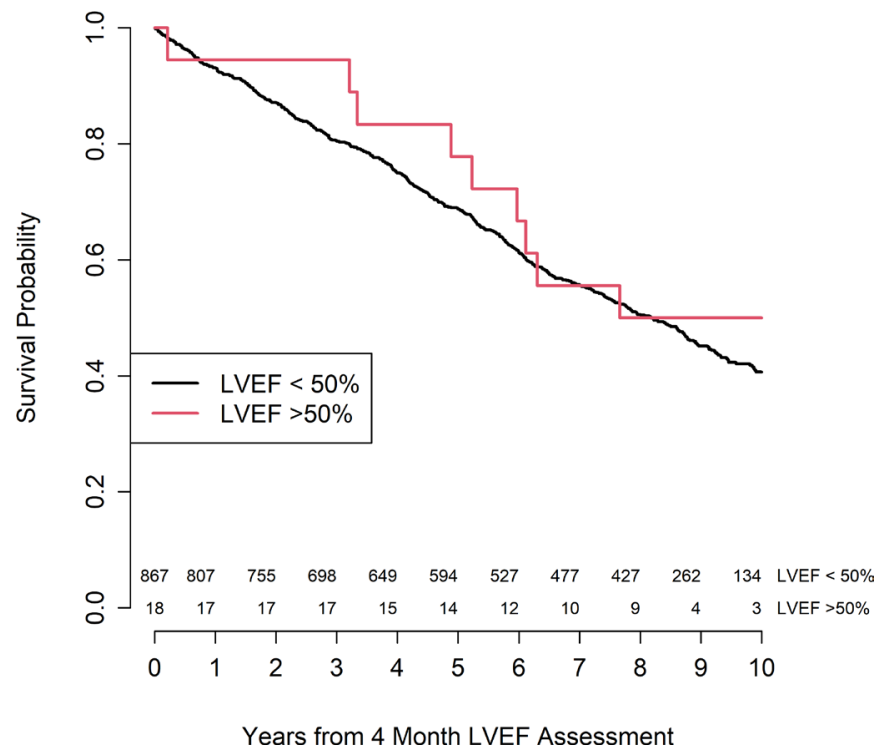

Figure 2 Association between LVEF recovery at 4 months and mortality survival probability for patients LVEF $>50 \%$ at 4 months is depicted in red, survival probability of patients with LVEF $<50 \%$ at 4 months is depicted in black. For each year mark on the $\mathrm{X}$-axis the number of subjects at risk in the two groups is reported above the axis, LVEF $<50 \%$ on top, LVEF $>50 \%$ on the bottom. Kaplan-Meier log-rank test $p=0.54$. LVEF, left ventricular ejection fraction.
Table 3 Relationship between LVEF recovery at 4 months and mortality

\begin{tabular}{llc}
\hline & HR $(\mathbf{C l})$ & P value \\
\hline LVEF recovery at 4 months & $0.93(0.48$ to 1.80$)$ & 0.82 \\
\hline Age, per decade & $1.37(1.24$ to 1.51$)$ & $<0.0001$ \\
Medical therapy vs CABG & $1.45(1.21$ to 1.73$)$ & $<0.0001$ \\
OMT & $0.82(0.68$ to 0.98$)$ & 0.031 \\
Diabetes & $1.29(1.08$ to 1.54$)$ & 0.006 \\
Female gender & $0.61(0.46$ to 0.82$)$ & 0.001 \\
BMI & $1.00(0.98$ to 1.02$)$ & 0.828 \\
\hline
\end{tabular}

Cox proportional hazard model of the relationship between LVEF recovery at 4 months and mortality during follow-up. For each variable, the HR for mortality during follow-up after adjusting for all other listed variables is indicated. $95 \% \mathrm{Cl}$ for the $\mathrm{HR}$ is reported in parentheses. Medical therapy vs CABG refers to patients randomised to medical therapy alone or to $C A B G$ plus medical therapy. OMT refers to patient on OMT at 4 months follow-up. $\mathrm{BMI}$, body mass index; CABG, coronary artery bypass graft; LVEF, left ventricular ejection fraction; OMT, optimal medical therapy.

landmark. These patients were less likely to be male $(85.5 \%$ vs $91.5 \%, \mathrm{p}=0.04$ ) but did not differ from the rest of the cohort with respect to other demographics, comorbidities and randomisation to OMT plus CABG or OMT alone (online supplemental table 2). Of these 634 patients, 35 were found to have recovered LVEF, while 599 were found to have reduced LVEF. Patients with recovered LVEF at 2 years were similar to patients with reduced LVEF in terms of age, BMI, prevalence of ICDs, prevalence of CRT, prevalence of diabetes and randomisation group within the STICH trial but were less likely to be male $(71.4 \%$ vs $86.3 \%$, $\mathrm{p}=0.03$, table 4$)$.

Table 4 Comparison between patients with and without LVEF recovery at 2 years

\begin{tabular}{lccl}
\hline & $\begin{array}{l}\text { LVEF } \leq \mathbf{5 0 \%} \\
\text { at 2 years } \\
\text { ( } \mathbf{n = 5 9 9 )}\end{array}$ & $\begin{array}{l}\text { LVEF }>\mathbf{5 0 \%} \\
\text { at 2 years } \\
\text { (n=35) }\end{array}$ & P value \\
\hline Age (SD) & $60.22(9.28)$ & $61.02(6.65)$ & 0.616 \\
\hline BMI (SD) & $27.48(4.54)$ & $27.46(6.01)$ & 0.977 \\
\hline $\begin{array}{l}\text { Medical therapy vs } \\
\text { CABG (\%) }\end{array}$ & $308(51.4)$ & $13(37.1)$ & 0.142 \\
\hline Male gender (\%) & $517(86.3)$ & $25(71.4)$ & 0.029 \\
\hline Diabetes (\%) & $242(40.4)$ & $20(57.1)$ & 0.075 \\
\hline CRT (\%) & $39(6.5)$ & $1(2.9)$ & 0.717 \\
\hline OMT (\%) & $404(67.4)$ & $26(74.3)$ & 0.512 \\
\hline ICD (\%) & $13(2.2)$ & $0(0.0)$ & 1 \\
\hline
\end{tabular}

Data presented as mean (SD) for continuous variables and number (percentage) for categorical variables. Patients with and without recovered LVEF at 2 years were compared using t-test for continuous variables and $\chi^{2}$ test for categorical variables. OMT refers to patient on OMT at 4 months follow-up.

BMI, body mass index; CABG, coronary artery bypass graft; CRT, cardiac resynchronisation therapy; ICD, permanent implanted cardiac defibrillator; LVEF, left ventricular ejection fraction; OMT, optimal medical therapy. 


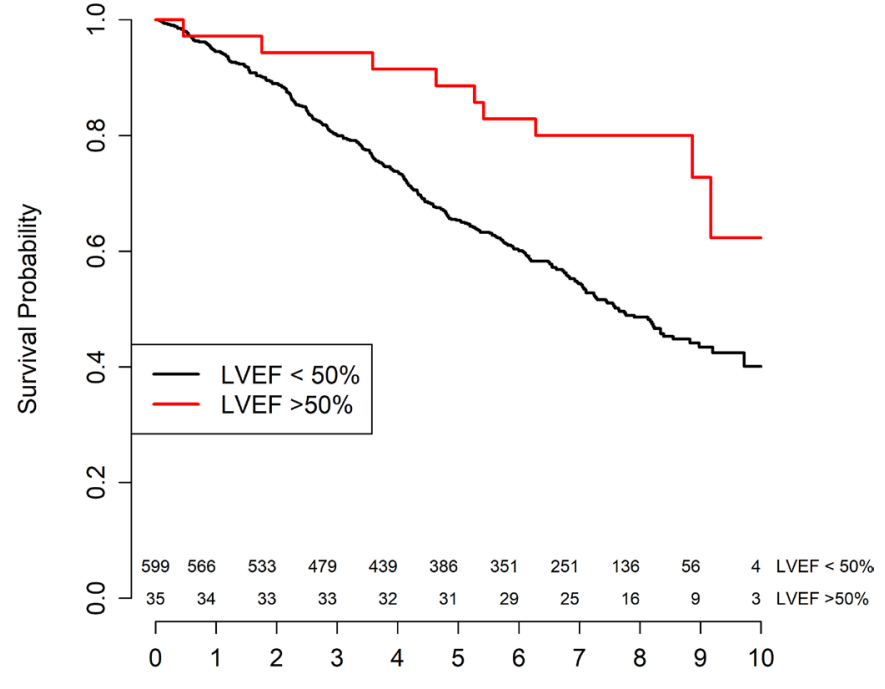

Years from 2-Year LVEF Assessment

Figure 3 Association between LVEF recovery at 2 years and mortality. survival probability for patients LVEF $>50 \%$ at 2 years is depicted in red, survival probability of patients with LVEF $<50 \%$ at 4 months is depicted in black. For each year mark on the X-axis the number of subjects at risk in the two groups is reported above the axis, LVEF $<50 \%$ on top, LVEF $>50 \%$ on the bottom. Kaplan-Meier log-rank test $p<0.01$. LVEF, left ventricular ejection fraction.

Recovery of LVEF at 2 years was associated with a reduction in all-cause mortality during follow-up, both in unadjusted survival analysis (log-rank $\mathrm{p}=0.004$, figure 3 ) and in the in the multivariate Cox proportional hazards model (HR: 0.41; 95\% CI: 0.21 to $0.80 ; \mathrm{p}=0.009$; table 5 ).

\section{DISCUSSION}

We performed a non-prespecified analysis of the STICH trial, the only contemporary randomised treatment trial

\begin{tabular}{llc}
\hline $\begin{array}{l}\text { Table } 5 \\
\text { and mortality }\end{array}$ & Relationship between LVEF recovery at 2 years \\
\hline & HR (CI) & P value \\
\hline LVEF recovery at 2 years & $0.41(0.21$ to 0.80$)$ & 0.009 \\
\hline Age, per decade & $1.36(1.20$ to 1.54$)$ & $<0.0001$ \\
\hline Female gender & $0.57(0.39$ to 0.83$)$ & 0.003 \\
\hline Diabetes & $1.24(0.99$ to 1.55$)$ & 0.066 \\
\hline Medical therapy vs CABG & $1.44(1.14$ to 1.80$)$ & 0.002 \\
\hline BMI & $1.00(0.97$ to 1.03$)$ & 0.947 \\
OMT & $0.95(0.74$ to 1.20$)$ & 0.652 \\
\hline
\end{tabular}

Cox proportional hazard model of the relationship between LVEF recovery at 2 years and mortality during follow. For each variable, the HR for mortality during follow-up after adjusting for all other listed variables is indicated. $95 \% \mathrm{Cl}$ for the $\mathrm{HR}$ is reported in parentheses. Medical therapy vs CABG refers to patients randomised to medical therapy alone or to CABG plus medical therapy. OMT refers to patient on OMT at 4 months follow-up. Statistical testing performed using Cox proportional hazard model. $\mathrm{BMI}$, body mass index; CABG, coronary artery bypass graft; OMT, optimal medical therapy. of patients with ischaemic heart failure. We found that in patients with ICM: (1) recovery of LVEF at short-term follow-up (4 months) does not correlate with recovery of LVEF at long-term follow-up(figure 1); (2) normalisation of LVEF in the short-term might not be a meaningful prognostic marker(figure 2, table 3); (3) Normalisation of LVEF at long-term (2 years) follow-up correlates with a reduced hazard of death (figure 3 , table 4 ). These findings expand our current understanding of LVEF normalisation in patients with ICM and are relevant to clinical practice.

Many patients with HFrEF of non-ischaemic aetiology experience a significant improvement in LVEF in the first year as a response to OMT. This improvement is typically an intermediate state before a subsequent deterioration and very seldom (if at all) marks the beginning of a permanent normalisation of LVEF. ${ }^{15}$ Patients with ICM tend to experience less improvement in LVEF than those with non-ischaemic disease, and seldom recover LVEF in the normal range ${ }^{5916}$ Data on LVEF trajectory in patients with normalised LVEF and ICM is nearly absent. In this study, we confirmed that normalisation of LVEF is a rare occurrence in patients with ICM. In addition, we found that while LVEF at short-term follow-up (4 months) following initiation of therapy correlated with LVEF at long-term follow-up (2 years), there was no correlation between early and late normalisation in LVEF. This is a novel and unexpected finding. LVEF is typically used as a surrogate of myocardial function, but is a measure that incorporates multiple cardiovascular factors, including preload, afterload (effective arterial elastance), inotropy (LV end-systolic elastance) and left ventricular stiffness. ${ }^{17}$ Accordingly, changes in different cardiovascular and haemodynamic parameters can result in an improvement in LVEF without an improvement in intrinsic myocardial contractile function. This is exemplified by the observation that treatment with negative inotropic agents such as beta-blockers can improve EF as early as 2 weeks after initiation of therapy. ${ }^{18}$ We therefore hypothesise that, in response to medical therapy, some patients with ICM may experience changes in several cardiovascular factors that affect LVEF, and that this might result in rapid, but ephemeral, improvement of LVEF to a normal range.

We found that normalisation in LVEF at 4 months was not associated with decreased mortality during follow-up, while normalisation in LVEF at 2 years was associated with mortality improvement. The observation of a correlation between recovered LVEF at 2 years and reduced mortality is novel because the literature reports no data on the prognostic significance of LVEF normalisation in patients with ICM, though it does parallel findings in cohorts of patients with non-ischaemic disease. $.^{3-7} 19-21$ The observation that rapid recovery in LVEF does not correlate with improved survival is both novel and unexpected insofar as normalisation in LVEF has been, to date, uniformly considered a positive prognostic factor. ${ }^{35}$ Patients with normalised LVEF at 4 months had a modest increase in the prevalence of diabetes of borderline statistical 
significance (table 2), but this is unlikely to explain our findings. We hypothesise that the increase in LVEF observed at short-term follow-up may result from changes in cardiovascular parameters, and therefore may not necessarily reflect the beneficial, progressive structural myocardial changes that have been classically associated with normalisation in LVEF and improved outcomes. ${ }^{22}$ Further work will be needed to explore this hypothesis. Until data are gathered, clinicians caring for patients with HFrEF of ischaemic origin should interpret rapid normalizations in LVEF with caution.

\section{Limitations}

A strength of this study is that it is the first to examine EF normalisation in patients with ICM. It is also the first to investigate the prognostic significance of normalised EF using clinical trial data. However, our work has several limitations that should be acknowledged. First, the echocardiographic measurements of LVEF collected in the STICH trial were not all obtained with the same method. It has been previously determined that the mean absolute differences between LVEF as determined by different echocardiographic methods in the STICH trial were minimal (mean absolute difference 2.7\% for biplane and visual, $3.0 \%$ for single plane and visual and $2.9 \%$ for biplane and single plane), ${ }^{23}$ but we cannot exclude the possibility that these small differences biased our findings. Second, normalisation of LVEF in patients with ICM is a rare event and therefore the number of patients with recovered LVEF that we analysed is small. These small numbers might reduce the reliability of our conclusions. Third, LVEF at 4 months was measured at 4 months from randomisation and not at 4 months from institution of therapy. ${ }^{24}$ Because some patients in the STICH trial experienced a delay between randomisation and initiation of therapy, ${ }^{11}$ the timing of this early assessment of LVEF normalisation might be inaccurate. Fourth, management of HFrEF has, to some extent, changed since the STICH trial and therefore our findings might not completely apply to contemporary patient cohorts. Fifth, while we know what classes of medications patients were on at 4 months postdischarge, we do not know the exact doses prescribed. Lastly, as with any non-prespecified secondary analysis of subgroup of patients within trial populations, our study should be considered as hypothesis generating and our findings will need to be confirmed in other studies.

\section{Conclusions}

In patients with HFrEF and ICM, normalisation of LVEF is a rare event. In this patient population, normalisation of LVEF soon after initiation of therapy did not correlate with sustained normalisation of LVEF and might not constitute a positive prognostic sign. Instead, normalisation of LVEF at 2 years after initiation of therapy correlated with a marked reduction in mortality risk during follow-up. Further studies will be needed to confirm these findings.
Twitter Walter B Schiffer @WalterBSchiffer, Andrew Perry @Perrycardium, David L Brown @DavidLBrownMD and Luigi Adamo @luigiadamomphd

Contributors All authors contributed to the design of the study. ED led the data analysis. All authors contributed to writing and reviewing the manuscript.

Funding Dr Adamo was supported by the NIH grant 1K08HL145108-01A1.

Competing interests None declared.

Patient consent for publication Not required.

Provenance and peer review Not commissioned; externally peer reviewed.

Data availability statement Data are available in a public, open access repository. Deidentified participant data from the STICH dataset was obtained from the NHLBI under a data use agreement. The Washington University Human Research Protection Office granted this study an exemption from Institutional Review Board oversight due to the deidentified nature of the data. This study complies with the Declaration of Helsinki.

Open access This is an open access article distributed in accordance with the Creative Commons Attribution Non Commercial (CC BY-NC 4.0) license, which permits others to distribute, remix, adapt, build upon this work non-commercially, and license their derivative works on different terms, provided the original work is properly cited, appropriate credit is given, any changes made indicated, and the use is non-commercial. See: http://creativecommons.org/licenses/by-nc/4.0/.

ORCID iDs

Walter B Schiffer http://orcid.org/0000-0002-2046-1993

David L Brown http://orcid.org/0000-0002-0930-9747

Luigi Adamo http://orcid.org/0000-0003-2704-978X

\section{REFERENCES}

1 Adamo L, Perry A, Novak E. Global longitudinal strain predicts sustained recovery of $L V$ ejection fraction in heart failure patients on evidence based medical therapies. J Am Coll Cardiol 2017;69:727.

2 Mann DL, Barger PM, Burkhoff D. Myocardial recovery and the failing heart: myth, magic, or molecular target? J Am Coll Cardiol 2012;60:2465-72.

3 Basuray A, French B, Ky B, et al. Heart failure with recovered ejection fraction: clinical description, biomarkers, and outcomes. Circulation 2014;129:2380-7.

4 Cioffi G, Stefenelli C, Tarantini L, et al. Chronic left ventricular failure in the community: prevalence, prognosis, and predictors of the complete clinical recovery with return of cardiac size and function to normal in patients undergoing optimal therapy. $J$ Card Fail 2004;10:250-7.

5 Lupón J, Díez-López C, de Antonio M, et al. Recovered heart failure with reduced ejection fraction and outcomes: a prospective study. Eur J Heart Fail 2017;19:1615-23.

6 Merlo M, Pyxaras SA, Pinamonti B, et al. Prevalence and prognostic significance of left ventricular reverse remodeling in dilated cardiomyopathy receiving tailored medical treatment. J Am Coll Cardiol 2011;57:1468-76.

7 Merlo M, Stolfo D, Anzini M, et al. Persistent recovery of normal left ventricular function and dimension in idiopathic dilated cardiomyopathy during long-term follow-up: does real healing exist? $J$ Am Heart Assoc 2015;4:e001504.

8 Givertz MM, Mann DL. Epidemiology and natural history of recovery of left ventricular function in recent onset dilated cardiomyopathies. Curr Heart Fail Rep;10:321-30.

9 Basuray A, Fang JC. Management of patients with recovered systolic function. Prog Cardiovasc Dis 2016;58:434-43.

$10 \mathrm{Klem}$ I, Shah DJ, White RD, et al. Prognostic value of routine cardiac magnetic resonance assessment of left ventricular ejection fraction and myocardial damage: an international, multicenter study. Circ Cardiovasc Imaging 2011;4:610-9.

11 Velazquez EJ, Lee KL, Deja MA, et al. Coronary-artery bypass surgery in patients with left ventricular dysfunction. $N$ Engl J Med 2011;364:1607-16.

12 Velazquez EJ, Lee KL, Jones $\mathrm{RH}$, et al. Coronary-artery bypass surgery in patients with ischemic cardiomyopathy. $N$ Engl J Med 2016;374:1511-20.

13 Velazquez EJ, Lee KL, Deja MA. Coronary-artery bypass surgery in patients with left ventricular dysfunction. New England Journal of Medicine 2011;364:1607-16.

14 Pellikka PA, She L, Holly TA, et al. Variability in ejection fraction measured by echocardiography, gated single-photon emission computed tomography, and cardiac magnetic resonance in patients 
with coronary artery disease and left ventricular dysfunction. JAMA Netw Open 2018;1:e181456-e56.

15 Perry AS, Mann DL. Brown DLImprovement of ejection fraction and mortality in ischaemic heart failureHeart (Published Online First: 25 August 2020).

16 Lupón J, Gavidia-Bovadilla G, Ferrer E, et al. Dynamic trajectories of left ventricular ejection fraction in heart failure. J Am Coll Cardiol 2018;72:591-601.

17 Monge García MI, Jian Z, Settels JJ, et al. Determinants of left ventricular ejection fraction and a novel method to improve its assessment of myocardial contractility. Ann Intensive Care 2019;9:48.

18 Maurer MS, Sackner-Bernstein JD, El-Khoury Rumbarger L, et al. Mechanisms underlying improvements in ejection fraction with carvedilol in heart failure. Circ Heart Fail 2009;2:189-96.

19 Florea VG, Rector TS, Anand IS, et al. Heart failure with improved ejection fraction: clinical characteristics, correlates of recovery, and survival: results from the valsartan heart failure trial. Circ Heart Fail 2016;9:e003123.
20 Chang K-W, Beri N, Nguyen NH, et al. Heart failure with recovered ejection fraction in African Americans: results from the AfricanAmerican heart failure trial. J Card Fail 2018;24:303-9.

21 de Groote P, Fertin M, Duva Pentiah A, et al. Long-Term functional and clinical follow-up of patients with heart failure with recovered left ventricular ejection fraction after $\beta$-blocker therapy. Circ Heart Fail 2014;7:434-9.

22 Adamo LM, Douglas L. Alterations in Ventricular Function: Role of Left Ventricular Remodelling and Reverse Remodelling in Heart Failure. In: DMaG F, ed. Heart Failure -A companion to Braunwald's Heart Disease. 3rd edn. Philadelphia: Elsevier, 2016: 178-94.

23 Mitchell C, Rahko PS, Blauwet LA. Guidelines for performing a comprehensive transthoracic echocardiographic examination in adults: recommendations from the American Society of echocardiography. J Am Soc Echocardiogr;32:1-64.

24 Velazquez EJ, Lee KL, O'Connor CM, et al. The rationale and design of the surgical treatment for ischemic heart failure (STICH) trial. $J$ Thorac Cardiovasc Surg 2007;134:1540-7. 